\title{
Méthode d'estimation du flux de chaleur dans les outils d'usinage
}

\author{
Jean-Luc Battaglia ${ }^{a}$, Ludivine Puigsegur, Haj El Moussami, Jean-Pierre Nadeau \\ et Jean-Christophe Batsale \\ Laboratoire «Énergétique et Phénomènes de Transfert» E.N.S.A.M., U.M.R. 8508, Esplanade des Arts et Métiers, \\ 33405 Talence Cedex, France
}

Reçu le 29 avril 2002, accepté le 23 octobre 2003

\begin{abstract}
Résumé - Nous proposons une méthode d'estimation du flux de chaleur dans un outil de coupe lors d'une opération d'usinage par tournage et fraisage. Le modèle du comportement thermique de l'outil, reliant ce flux à la température mesurée en un point de l'outil, est obtenu au sens de l'identification de système non entier. Nous réalisons pour cela un dispositif de mesure de la température dans l'outil ainsi qu'un banc expérimental de caractérisation permettant de mesurer et de contrôler le flux de chaleur appliqué sur l'outil. Le modèle obtenu, après la phase d'identification des paramètres, permet de calculer la réponse impulsionnelle. Cette réponse est alors utilisée dans une procédure d'estimation séquentielle du flux.
\end{abstract}

Mots clés : Usinage / flux de chaleur / problème inverse / identification de système non entier / métrologie thermique

\begin{abstract}
Heat fluxes estimation in manufacturing tools. We propose estimating heat flux in tools during machining by turning and milling. The thermal behaviour model of the tool, which rely the heat flux and the temperature at the sensor, is achieved from the non integer system identification approach. An apparatus is realized in order to measure the temperature at a point in the tool. Another apparatus for the tool characterization permit controlling and measuring the heat flux applied on the tool. The impulse response is computed from the identified model and used in a sequential procedure to estimate the heat flux in the tool during machining.
\end{abstract}

Key words: Manufacturing / heat flux / inverse problem / non integer system identification / temperature measurement

\section{Introduction}

Cette étude est dédiée à l'estimation du flux de chaleur dans les outils de coupe utilisés durant les procédés d'usinage de tournage et de fraisage. La méthode s'applique indifféremment quelque soit la nature du matériau usiné, métallique ou non. Une description des méthodes utilisées jusqu'en 1973 est présentée dans les travaux de Barrow [1]. Grâce aux moyens de calculs de plus en plus performants et à l'évolution technologique des dispositifs de mesure de température ces méthodes ont encore évolué depuis.

Une première catégorie de méthodes, que nous qualifions de directes, consiste à mesurer directement la température moyenne à l'interface entre l'outil et le matériau usiné. La méthode la plus connue utilise le thermocouple naturel formé par la pièce et l'outil lorsque les deux matériaux sont conducteurs de l'électricité, voir par

\footnotetext{
a Auteur correspondant : jlb@lept-ensam.u-bordeaux.fr
}

exemple les premiers travaux de Shore [2] et Herbert [3] dans ce domaine et ceux plus récents de Stephenson [4]. C'est une mesure moyenne en ce sens qu'elle intègre toute la surface de contact entre le copeau et la pièce, donc la zone de cisaillement secondaire ainsi que les deux zones en dépouille. Cette température évoluant fortement sur toute la longueur du contact, la température maximale atteinte sur l'outil dans la zone de cisaillement secondaire ne sera pas accessible à partir de cette mesure. Le matériau usiné est d'autre part en mouvement relatif par rapport à l'outil. Il existe donc, de par la nature imparfaite de contact, une résistance thermique de contact glissant fonction principalement des paramètres de coupe et de la nature des matériaux. Ainsi, la température moyenne mesurée à l'interface est différente de la température moyenne à la surface de l'outil et de la température moyenne à la surface de la matière usinée. Signalons enfin que la mesure directe nécessite un appareillage expérimental délicat du fait des mouvements de rotation de l'outil, dans le cas du fraisage, ou de la pièce dans le cas du tournage. Ainsi, 
la continuité du signal électrique doit être généralement assurée au travers de roulements mercure qui permettent de limiter le phénomène d'auto échauffement.

La deuxième catégorie de méthodes est basée sur une mesure dite indirecte ou inverse. La démarche consiste à estimer le flux de chaleur dans l'outil, à partir de mesures de température réalisées en un ou plusieurs points de l'outil, éloignés de quelques millimètres de la zone de cisaillement secondaire. Il est alors nécessaire de construire un modèle du comportement thermique transitoire de l'outil liant le flux à estimer aux mesures de température des capteurs. Les travaux de Lipman et al. [5], Groover et Kane [6], Yen et Wright [7], Stephenson [8], Lin [9], El-Wardany et al. [10] s'intéressent directement à l'estimation de la température moyenne à l'interface outilcopeau. De par la variation spatiale de température à l'interface et l'incertitude sur la mesure de l'aire de contact glissant, le domaine de confiance associé à l'estimation de cette température s'avère très étroit. Il est donc plus judicieux d'estimer le flux de chaleur dans l'outil puis de recalculer le champ thermique sur l'aire de contact en modélisant le transfert de chaleur dans le bec d'outil à partir d'une méthode de type éléments finis par exemple. Cette approche est d'ailleurs utilisée dans les récents travaux de Chen et al. [11], Kwon et al. [12], Olson et Throne [13] et Lima et al. [14] qui présentent différentes approches, en termes de dispositifs métrologiques et de modélisation, pour l'estimation du flux de chaleur dans les outils d'usinage en tournage.

Notre démarche repose elle aussi sur la résolution du problème inverse de diffusion de la chaleur dans l'outil pour estimer le flux de chaleur. La mesure de température est réalisée en un seul point de l'outil. La différence principale, au regard des différents travaux présents dans la littérature et cités précédemment, est liée à la formulation du modèle de diffusion de la chaleur dans l'outil. En effet, la modélisation à partir des équations aux dérivées partielles de la thermique : conservation de l'énergie et conditions aux limites, nécessite tout d'abord que l'on connaisse avec précision l'ensemble des paramètres thermophysiques, diffusivité et conductivité thermiques, des différents matériaux constitutifs de l'outil ainsi que les coefficients d'échange prenant en compte la convection et le rayonnement aux frontières. Ceci suppose donc qu'il est préalablement nécessaire d'effectuer autant d'expériences de caractérisation thermique adaptées à l'identification des paramètres inconnus. Il est d'autre part nécessaire de connaître avec précision la morphologie de l'outil, à savoir l'imbrication des différents éléments constitutifs et le positionnement des points de contact. On note que cette complexité géométrique nécessite l'utilisation de méthodes de discrétisation spatiale telle que la méthode des éléments finis en vue de la résolution des équations aux dérivées partielles. Enfin, la résolution du problème inverse est très sensible à la précision avec laquelle on va localiser les capteurs de températures dans le maillage généré. Comme nous le verrons par la suite, ceci représente un véritable problème lorsque les dimensions du capteur sont du même ordre de grandeur que la distance entre le capteur et la zone où est appliqué le flux, ce qui est le cas des thermistances.

Devant une telle problématique, il nous a semblé intéressant d'élaborer le modèle du comportement thermique transitoire de l'outil au sens de l'identification des systèmes. Nous proposons donc d'identifier les paramètres d'un modèle liant des mesures de température aux capteurs à celles du flux de chaleur dans l'outil. Comme nous l'avons montré dans de précédents travaux [15-17], le modèle le plus cohérent vis-à-vis de la nature du transfert étudié, ici la diffusion, est représenté sous la forme d'une loi exprimant les dérivées fractionnaires, d'ordre multiple de $1 / 2$, du flux dans l'outil et de la température aux capteurs. Ainsi, les paramètres de ce modèle sont identifiés en reproduisant les mêmes conditions thermiques appliquées sur l'outil que celles rencontrées durant l'usinage. Cette étape constitue la difficulté principale d'une telle approche car elle nécessite la réalisation d'un banc expérimental de caractérisation de l'outil. Il est par contre clairement établi que le flux estimé lors de l'usinage à partir du modèle identifié possède un domaine de confiance beaucoup plus important que lorsque l'on utilise le modèle aux EDP classiques où certains paramètres thermophysiques sont estimés avec une erreur importante. En effet, la caractérisation au sens de l'identification de système nécessite au plus deux expériences, la deuxième servant de validation au système identifié lors de la première. D'autre part, le système identifié prend en compte la dynamique du capteur ainsi que son éventuelle influence sur le transfert de chaleur dans l'outil. Ce même capteur étant utilisé pour l'estimation du flux durant l'usinage, la connaissance de sa localisation exacte dans l'outil n'est donc plus nécessaire.

Dans un premier temps, nous exposons la méthode mathématique d'estimation du flux de chaleur à partir de la mesure de température en un point de l'outil et de la réponse impulsionnelle du système. Dans un deuxième temps, nous discutons du choix de représentation du modèle de diffusion de la chaleur dans l'outil sous la forme d'un modèle non entier liant les dérivées fractionnaires successives de la température au capteur et du flux dans l'outil. Nous présentons ensuite les différents appareillages métrologiques que nous avons développés pour les procédés d'usinage par tournage et fraisage. Nous présentons enfin quelques résultats obtenus dans deux configurations d'usinage très différentes.

\section{Méthode d'estimation du flux de chaleur dans l'outil}

Nous utilisons la technique séquentielle d'estimation avec spécification de fonction constante décrite dans l'ouvrage de Beck [18]. Soit $\Delta t$ la période d'échantillonnage des mesures. La méthode consiste à estimer le flux $\hat{\phi}(t)$ dans l'outil à l'instant $t$ à partir des mesures de température $Y_{P}(t)$ au capteur depuis l'instant $t$ jusqu'à l'instant $(t+r \Delta t)$ et des valeurs du flux estimé aux instants précédents. On suppose que le flux reste 
constant durant l'intervalle $r \Delta t, r$ étant un nombre entier généralement compris entre 2 et 4 , de façon à résoudre un système surdéterminé au sens des moindres carrés linéaires. Soit $h(t)$ la réponse impulsionnelle liant la température $T_{P}(t)$ au capteur et le flux de chaleur $\phi(t)$ dans l'outil à partir du produit de convolution :

$$
T_{P}(t)=\int_{0}^{t} h(\tau) \phi(t-\tau) \mathrm{d} \tau
$$

La réponse impulsionnelle $h(t)$, correspondant à la réponse à un Dirac du flux $\delta[\phi(t)]$, est calculée à partir du modèle de diffusion de la chaleur dans l'outil, modèle qui est établi par ailleurs. La relation de convolution (1) suppose que ce modèle est linéaire, soit donc que les propriétés thermophysiques du matériau sont indépendantes de la température. Cette hypothèse peut être mise en défaut au voisinage immédiat de la zone de coupe. Néanmoins, le gradient de température est très grand et le domaine de forte non linéarité reste petit en regard de la distance entre la zone sollicitée et le capteur, ce qui rend légitime l'hypothèse de linéarité.

La forme discrète du produit de convolution s'écrit :

$$
T_{P}(k \Delta t)=\sum_{l=0}^{k} h(l \Delta t) \phi((k-l) \Delta t)
$$

On montre alors que l'estimation du flux $\hat{\phi}(k \Delta t)$ à l'instant $t=k \Delta t$ est donnée par :

$$
\hat{\phi}(k \Delta t)=\frac{\sum_{j=0}^{r}\left(Y_{P}((k+j) \Delta t)-\tilde{T}((k+j) \Delta t)\right) h(j \Delta t)}{\sum_{j=0}^{r} h(j \Delta t)^{2}}
$$

avec :

$$
\tilde{T}((k+j) \Delta t)=\sum_{l=1}^{k+j} h(l \Delta t) \hat{\phi}((k+j-l) \Delta t)+T_{P}(0)
$$

Dans cette dernière relation $T_{P}(0)$ représente la température initiale du capteur. De par sa simplicité, la relation (3) permet l'estimation du flux dans l'outil durant l'usinage, donc en temps réel.

\section{Modèle du comportement thermique transitoire de l'outil}

\subsection{Pourquoi identifier un modèle de comportement?}

Cette approche se justifie tout d'abord au regard de la complexité de l'outil, en termes de nombre d'éléments constitutifs et de leurs imbrications mutuelles. Comme nous l'avons signalé dans l'introduction, une modélisation classique aux sens des équations aux dérivées partielles de la thermique nécessiterait l'estimation des propriétés thermiques des matériaux constitutifs de chaque élément ainsi que des résistances thermiques de contact à chaque interface. D'autre part, les caractéristiques géométriques du capteur de température et sa position vis-à-vis de la surface sollicitée peuvent nécessiter de prendre en compte son influence spatio-temporelle éventuelle sur le transfert de chaleur dans l'outil.

L'approche par identification de système, présentée dans les ouvrages de référence de Ljung [19] et Söderstrom [20], consiste à identifier les paramètres d'une loi liant le flux appliqué à l'outil et la température du capteur à partir de mesures de ces deux grandeurs. Il est donc nécessaire de pouvoir contrôler et mesurer le flux appliqué sur la face de coupe de l'outil. Cette expérience de caractérisation de l'outil doit nécessairement se dérouler dans des conditions identiques à celles rencontrées durant le procédé d'usinage. En d'autres termes de dispositif de chauffe générant le flux de chaleur dans l'outil ne doit ni occulter ni engendrer des comportements dynamiques autres que ceux propres à l'outil en situation d'usinage. Ceci revient à considérer que le dispositif de chauffe doit nécessairement avoir une inertie thermique petite devant le temps de diffusion de la chaleur entre la zone chauffée et le capteur.

\subsection{Identification d'une structure de modèle cohérente avec le phénomène de diffusion de la chaleur}

La structure de modèle classique, généralement utilisée en traitement du signal, exprime les dérivées successives par rapport au temps du flux en fonction de celles de la température au capteur. Une application dans le domaine de la thermique de l'usinage est présentée dans [21]. Dans des configurations 1D simples, où les solutions analytiques sont connues, cette formulation est insatisfaisante car un nombre très élevé de paramètres est nécessaire pour retrouver le comportement asymptotique aux temps courts. En recherchant une représentation de ces solutions analytiques cohérente avec les comportements asymptotiques aux temps courts et aux temps longs et assurant leur raccordement, nous avons abouti à une relation liant le flux de chaleur à la température au capteur sous la forme suivante :

$$
\sum_{i=0}^{M} a_{i} D^{i / 2} T_{p}(t)=\sum_{i=0}^{L} b_{i} D^{i / 2} \phi(t), \text { avec } a_{0}=1
$$

On pourra se référer aux publications $[15,16]$ pour les démonstrations. La relation (5) se distingue essentiellement de la relation classiquement utilisée en identification de systèmes par le fait que les ordres de dérivation sont réels et en particulier multiples de $1 / 2$, le symbole $\mathrm{D}^{\nu}$ désignant l'opérateur de dérivation fractionnaire d'ordre $\nu$ par rapport au temps. Le couple $(M, L) \in N^{2}$ caractérise la complexité du modèle, fonction principalement de la position $P$ du capteur vis-à-vis de la surface chauffée. 
Rappelons que la dérivée non entière d'ordre $\nu$ est explicitement définie par :

$$
\mathrm{D}^{\nu} f(t)=\mathrm{D}^{n} \mathrm{I}^{n-\nu} f(t)
$$

$n$ est l'entier directement supérieur ou égal à $\nu$ et $\mathrm{I}^{\nu}$ désigne l'intégrale fractionnaire d'ordre $\nu$ définie, au sens de Riemann Liouville, par :

$$
\mathrm{I}^{\nu} f(t)=\frac{1}{\Gamma(\nu)} \int_{0}^{t}(t-u)^{\nu-1} f(u) \mathrm{d} u
$$

$\Gamma$ est la fonction Eulérienne de deuxième espèce. Le lecteur pourra se référer aux ouvrages de références [22-25] concernant le calcul fractionnaire pour retrouver l'ensemble des définitions et propriétés liées à cet opérateur.

Des simulations numériques, voir [17], montrent que l'on peut généraliser la relation (5) à la modélisation du processus de diffusion de la chaleur dans les configurations $2 \mathrm{D}$ et $3 \mathrm{D}$. Ce modèle sera donc représentatif du processus de diffusion de la chaleur dans l'outil étudié.

L'objectif de l'identification de système est d'estimer le vecteur des paramètres $\theta=\left[\begin{array}{ll}a_{1} \cdot a_{M} & b_{0} \cdot b_{L}\end{array}\right]^{T}$ à partir de mesure du flux $\phi(t)$ et de la température $Y_{P}(t)$ au capteur. Cette identification repose sur l'utilisation des moindres carrés linéaires lorsque l'on cherche à minimiser l'erreur d'équation. Malheureusement, dans ce cas, l'estimation est biaisée et il est nécessaire d'utiliser la méthode des variables d'états pour minimiser ce biais. Les principales étapes de ce calcul d'optimisation linéaire sont rappelées en annexe.

\section{Application de la méthode à l'estimation des flux de chaleur dans les outils de tournage et de fraisage}

\subsection{Présentation des différents dispositifs métrologiques}

\subsubsection{Présentation du dispositif de mesure de température dans l'outil de tournage}

Le capteur est constitué par une thermistance, diamètre $0,45 \mathrm{~mm}$ et longueur $3 \mathrm{~mm}$, dont le temps de réponse est d'environ $100 \mathrm{~ms}$. La sensibilité de ce capteur, de l'ordre du $\mathrm{mV} /{ }^{\circ} \mathrm{C}$, permet d'obtenir un rapport signal sur bruit élevé et ne nécessite pas d'amplification. Comme cela est représenté sur la figure 1, le capteur est disposé dans la cale support, à une distance $e$ du bec d'outil variant entre 3 et $5 \mathrm{~mm}$ selon le type de plaquette utilisé. Le logement du capteur est réalisé par procédé d'électroérosion permettant d'obtenir un canal de diamètre $0,5 \mathrm{~mm}$. Le maintien de la thermistance en position fixe dans le canal est assuré par une colle thermodurcissable base argent. Le domaine d'utilisation du capteur, compris entre -50 et $150{ }^{\circ} \mathrm{C}$, est respecté de par sa position vis-à-vis du bec d'outil.

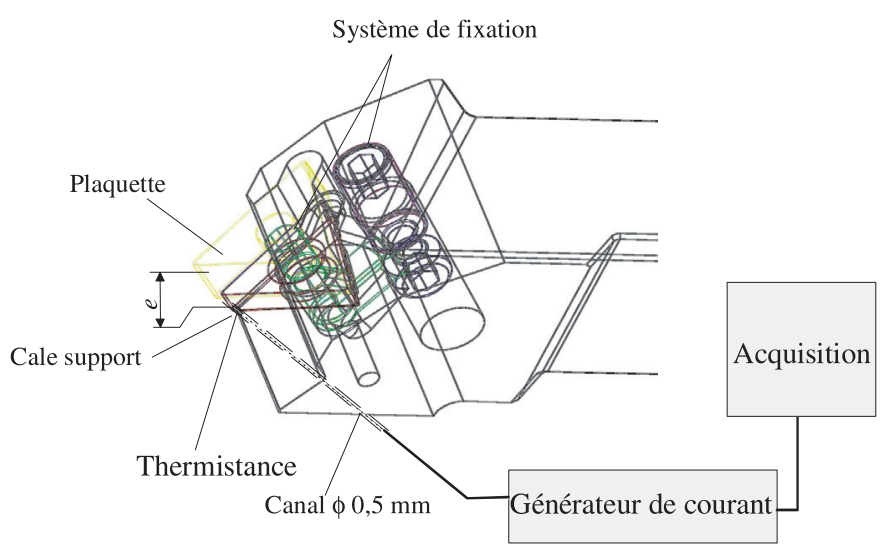

Fig. 1. Dispositif métrologique de mesure de la température en un point de l'outil de tournage. La thermistance est intégrée dans la cale support par l'intermédiaire d'un canal réalisé en électroérosion. L'extrémité du capteur est à la distance $e$ du bec de plaquette ( $e$ varie entre 3 et $5 \mathrm{~mm}$ suivant le type de plaquette).

On constate que, conformément à la situation envisagée au chapitre précédent, les dimensions du capteur sont du même ordre de grandeur que la distance entre son extrémité et le bec de plaquette, soit environ $3 \mathrm{~mm}$. La mesure de température n'est donc pas ponctuelle et le capteur joue un rôle non négligeable sur le transfert de chaleur dans l'outil dans la zone de mesure.

\subsubsection{Présentation du dispositif de mesure de température dans un outil de fraisage}

Lorsque les outils ont un mouvement de rotation par rapport à la pièce usinée, le dispositif de mesure thermique classiquement utilisé dans la littérature consiste à associer un thermocouple et une bague de roulement remplie de mercure pour éviter le phénomène d'auto échauffement. Néanmoins, la mesure dans les conditions réelles d'usinage reste fortement perturbée du fait de la très faible sensibilité du capteur (quelques $\mu \mathrm{V}$ par ${ }^{\circ} \mathrm{C}$ ). D'autre part, la mise en œuvre d'un tel dispositif est délicate et coûteuse. Ainsi, comme précédemment, nous avons utilisé des thermistances dont la sensibilité est 1000 fois supérieure à celle des thermocouples. La liaison entre les thermistances et le dispositif d'acquisition est alors simplement assurée par un collecteur tournant à vitesse de rotation élevée, jusqu'à $10000 \mathrm{tr} \cdot \mathrm{min}^{-1}$. Un tel dispositif a été utilisé dans les travaux de Broussely [26] pour la mesure de température dans un moteur asynchrone. Nous montrons, sur la figure 2, le dispositif de mesure dans sa globalité. Chaque thermistance est placée dans le corps de fraise au plus près de l'arête de coupe de chaque plaquette. Les logements des thermistances sont réalisés par procédé d'électroérosion et le maintien en position est réalisé par une colle thermodurcissable base argent. 


$$
\begin{gathered}
{\left[\begin{array}{cc}
0,7 \mathrm{D}^{-1 / 2} \\
{\left[ \pm 0,476 \times 10^{-4}\right]+\left[ \pm 0,103 \times 10^{-4}\right]}
\end{array} \begin{array}{c}
0,017 \mathrm{D}^{-3 / 2} \\
{\left[ \pm 1 \times 10^{-8}\right]}
\end{array}\right] T_{P}(t)=} \\
{\left[\begin{array}{ccc}
0,1823 & 1,223 \mathrm{D}^{-1 / 2} & 1,653 \mathrm{D}^{-1} \\
{\left[ \pm 0,025 \times 10^{-3}\right]} & -\left[ \pm 0,219 \times 10^{-3}\right]+\left[ \pm 0,163 \times 10^{-3}\right]
\end{array}\right] \phi(t)}
\end{gathered}
$$

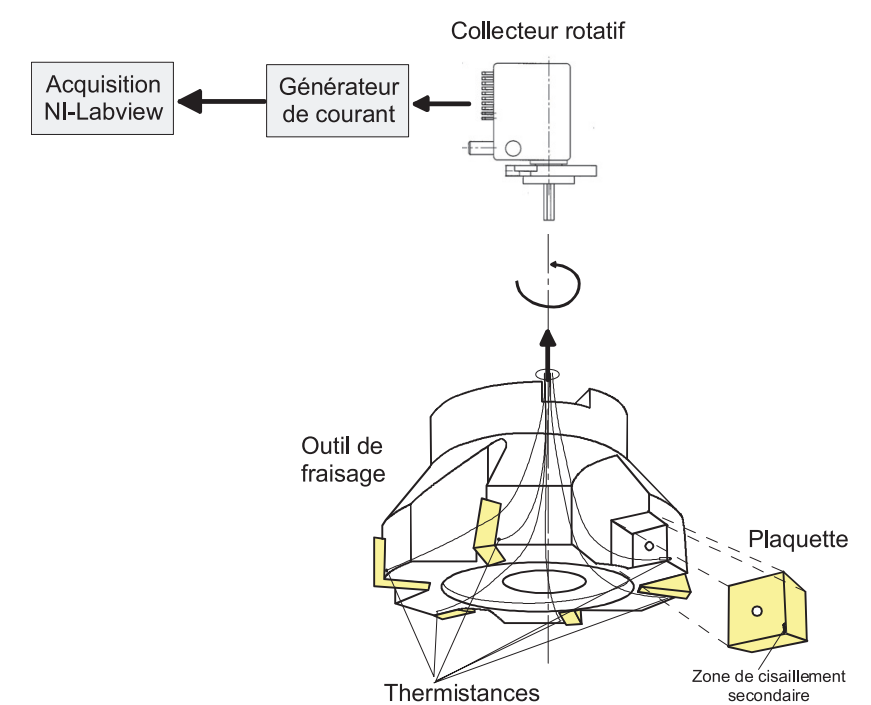

Fig. 2. Dispositif de mesure de température en différents points d'un outil de fraisage. Une thermistance est intégrée dans le corps de fraise derrière chaque plaquette (la distance entre le bec de plaquette et l'extrémité de la thermistance est $e=3 \mathrm{~mm}$ ). L'acquisition des signaux issus des thermistances est réalisée via un collecteur tournant grande vitesse à paliers.

\subsubsection{Présentation du dispositif expérimental de caractérisation thermique des outils}

Le dispositif de chauffe du banc de caractérisation est représenté sur la figure 3 . Il est constitué par une micro résistance en platine sérigraphiée sur une plaquette d'alumine d'épaisseur $250 \mu \mathrm{m}$ et de dimensions 4,6 $\mathrm{mm} \times$ $3,6 \mathrm{~mm}$. Ce composant est collé sur la face de coupe de la plaquette de l'outil à l'aide d'une colle thermodurcissable base argent (il est évidemment retiré une fois la caractérisation thermique de l'outil réalisée). Les petites dimensions de la micro résistance et l'arrangement tronconique de la colle permettent un chauffage local, correspondant approximativement à la surface de contact entre le copeau et l'outil durant l'usinage. Comme nous l'avons signalé en introduction, il est important de noter qu'une petite variation de la surface chauffée, entre les phases de caractérisation et d'usinage, n'affecte pas l'estimation du flux de chaleur réellement appliqué sur l'outil durant l'usinage étant donné la localisation de la thermistance vis-àvis du bec de plaquette. L'inertie thermique du système de chauffage n'excédant pas 0,1 seconde, il ne modifie donc quasiment pas le comportement thermique dynamique de l'outil. De plus, notons que la période d'échantillonnage des données pour la caractérisation est $\Delta t=0,2 \mathrm{~s}$. En négligeant les pertes, on admet donc que le flux de chaleur dans l'outil est égal à la puissance électrique fournie à la micro résistance.

\subsection{Identification du comportement thermique d'un outil de tournage}

La figure 4a présente les données générées pour l'identification du modèle liant le flux dans l'outil à la température au capteur lors de la caractérisation d'un outil de tournage. Afin d'améliorer l'identification aux temps courts, on génère une succession de créneaux de flux dont la largeur est petite devant la durée totale de l'essai. Il est à noter que la température ambiante reste constante durant toute la durée de l'essai et que $T_{P}(t)$ représente en fait l'écart entre la température au capteur et la température ambiante. Afin de minimiser l'influence de l'erreur de mesure lors de l'identification, il est préférable d'effectuer l'intégration plutôt que la dérivation des signaux (cf. annexe). Le modèle identifié obtenu, à partir de la méthode décrite en annexe, est :

\section{Voir équation (8) ci-dessus.}

On compare, sur la même figure 4a, la mesure de température avec la réponse du modèle identifié en considérant les mesures de flux généré expérimentalement et on vérifie la bonne superposition des deux courbes. En pratique, il est nécessaire de valider ce modèle en considérant d'autres mesures de flux et de température. Cette validation est représentée sur la figure $4 \mathrm{~b}$ et elle confirme donc bien la fiabilité du modèle identifié lors du premier essai.

\subsection{Estimation du flux de chaleur dans un essai de tournage - Application à l'analyse de l'influence de revêtements d'outil}

Nous présentons une application consistant à estimer le flux de chaleur dans un outil de tournage lors du dressage de la face d'un disque de la périphérie vers le centre tel que cela est représenté sur la figure 5 . Nous effectuons 6 essais d'usinage en conservant à chaque fois les mêmes paramètres de coupe. Dans le premier essai l'outil est muni d'une plaquette en carbure classique et c'est celui qui a été caractérisé au paragraphe précédent. Pour les 5 essais suivants, nous considérons le 


$$
\begin{aligned}
& {\left[\begin{array}{ccccc}
0,691 \mathrm{D}^{-1 / 2} & 0,609 \mathrm{D}^{-1} & 0,121 \mathrm{D}^{-3 / 2} & 0,024 \mathrm{D}^{-2} & 0,007 \mathrm{D}^{-5 / 2} \\
1- & {[ \pm 0,013]}
\end{array}+{ }_{[ \pm 0,009]}-{ }_{[ \pm 0,005]}+{ }_{[ \pm 0,002]}+\underset{[ \pm 0,012]}{[ \pm 0}\right] T_{P}(t)=}
\end{aligned}
$$

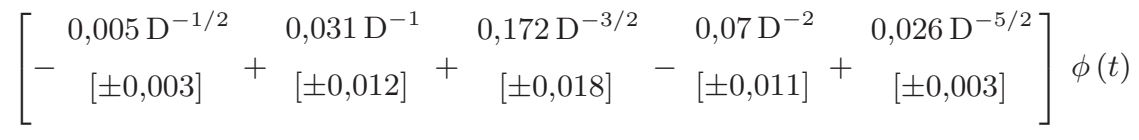

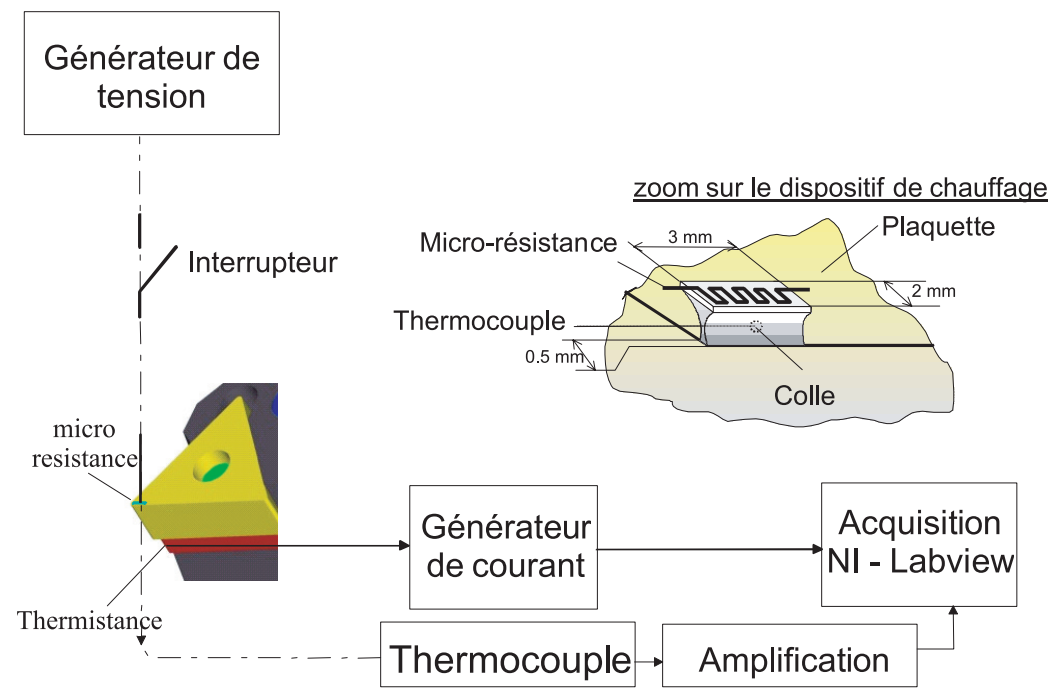

Fig. 3. Dispositif expérimental de caractérisation thermique des outils (représentation sur un outil de tournage). Le flux de chaleur est généré par une micro résistance collée sur la face de coupe de la plaquette. Un interrupteur permet de générer un flux transitoire sous la forme d'une séquence binaire pseudo aléatoire riche en hautes fréquences.

même outil mais avec des revêtements différents : $\mathrm{Al}_{2} \mathrm{O}_{3}$, TiN, TiAlN, TiAlNwc et TiAlN $+\mathrm{MoS}_{2}$. Ces revêtements, d'épaisseur 2 à $3 \mu \mathrm{m}$, n'ont aucune influence sur la comportement thermique intrinsèque à l'outil pour la période d'échantillonnage ( $0,2 \mathrm{~s})$ et la durée de l'essai (18 s) considérées. En d'autres termes, la réponse impulsionnelle pour chaque outil, utilisée dans la relation d'estimation du flux (3), est la même et correspond à celle de l'outil non revêtu. Cette réponse impulsionnelle est calculée à partir du modèle représenté par la relation (8). Sur la figure 6 , nous représentons la différence entre le flux estimé dans l'outil non revêtu et celui estimé dans chaque outil revêtu. Ces résultats mettent en évidence l'influence significative du revêtement $\mathrm{Al}_{2} \mathrm{O}_{3}$ pour le couple outil-matière considéré.

\subsection{Identification du comportement thermique d'un outil de fraisage}

Lorsque l'on chauffe l'arête de coupe d'une seule plaquette, on constate que, sur la durée de l'expérience, seule la température mesurée par le capteur associé à la plaquette chauffée est sensible à la variation de flux dans la plaquette (voir l'essai sur la Fig. 7). De plus, comme l'outil de fraisage est axisymétrique, nous admettons donc que chaque couple \{plaquette-capteur\} se comporte comme un système mono variable indépendant des autres. En d'autres termes, le modèle liant la température d'un capteur au flux de chaleur dans la plaquette associée est le même pour chaque couple \{plaquette-capteur\}. La figure 7 présente les données générées pour l'identification du modèle, soit donc le flux de chaleur dans la plaquette et la température au capteur correspondante. La durée d'acquisition des données est égale à 80 secondes et la période d'échantillonnage est égale à 0,02 seconde. Afin d'améliorer l'identification aux temps courts, on génère une succession de créneaux de flux de chaleur sur l'outil.

On trouve que le modèle qui permet de faire correspondre au mieux la température simulée à celle mesurée par le capteur est le suivant :

\section{Voir équation (9) ci-dessus.}

Sur la même figure 7 , nous montrons que la température calculée à partir du modèle précédent pour la variation de flux mesurée s'ajuste à la température mesurée au capteur.

\subsection{Estimation du flux de chaleur dans un essai de fraisage}

Nous réalisons un essai de surfaçage par fraisage tel que représenté sur la figure 8 . Nous montrons sur la figure 9 les résultats d'estimation du flux dans chaque plaquette durant cet essai d'usinage. Comme précédemment, 


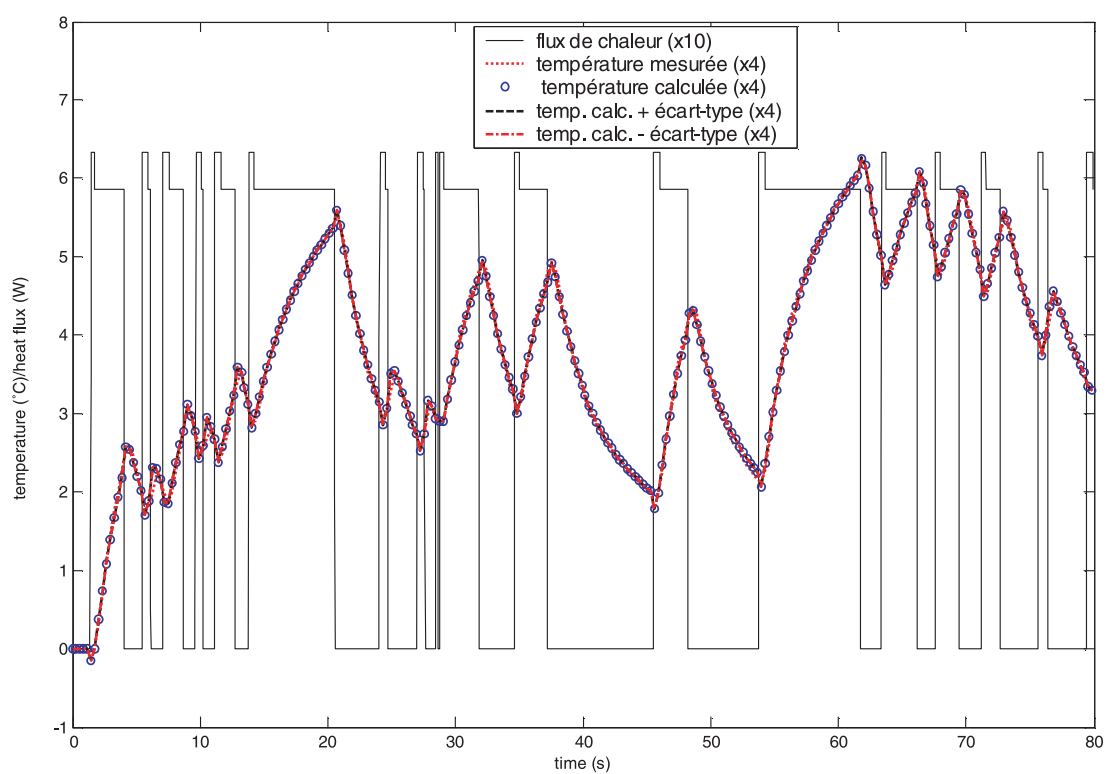

(a)

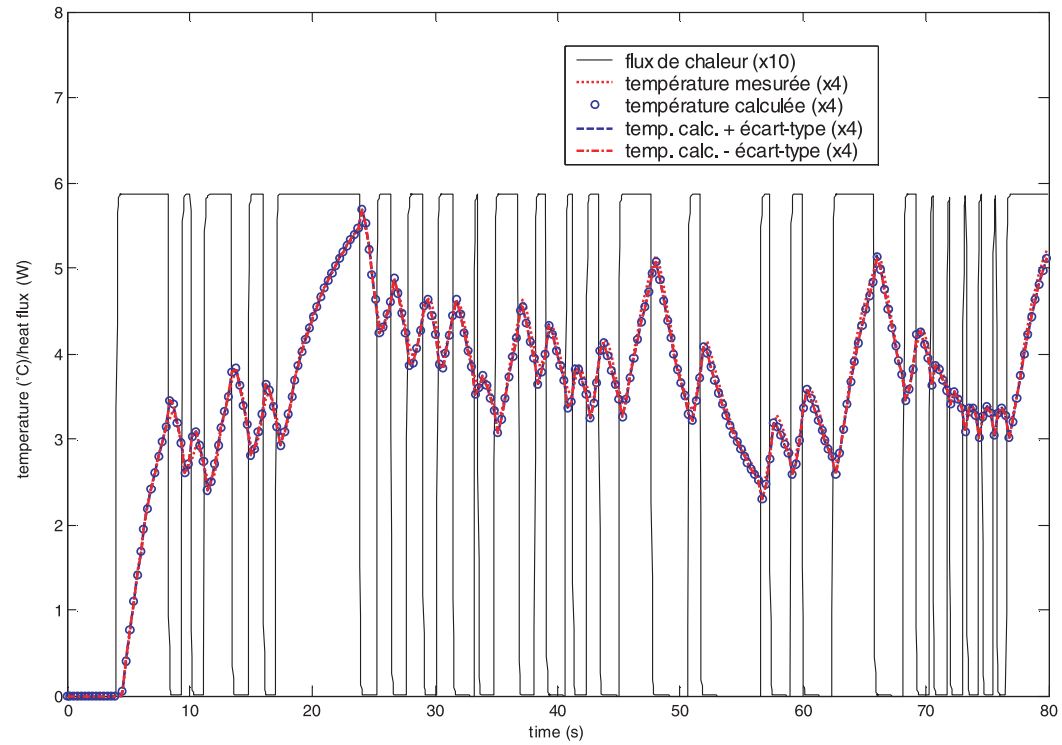

(b)

Fig. 4. (a) Mesures de flux dans l'outil et de température au capteur relevées durant une expérience de caractérisation d'un outil de tournage. On constate la très bonne adéquation entre les mesures au capteur et celles simulées à partir du modèle identifié. On représente l'écart type sur la température calculée à partir des écarts types sur les paramètres identifiés; (b) validation du système identifié à partir d'un second essai. On représente l'écart type sur la température calculée à partir des écarts types sur les paramètres identifiés.

nous utilisons la relation d'estimation (3) où la réponse impulsionnelle est calculée à partir du modèle non entier identifié précédemment. La figure 9 montre bien les deux phases caractéristiques du procédé, à savoir la phase d'engagement de la fraise dans la matière durant laquelle le flux évolue continûment et la phase où toute la fraise est totalement engagée et où le flux dans chaque plaquette est quasiment constant. Les résultats obtenus montrent également le fonctionnement différent de chaque plaquette de la fraise caractérisé par des variations importantes des niveaux de flux.

\section{Conclusion}

Nous avons développé une méthode d'estimation du flux de chaleur dans les outils d'usinage de matériaux métalliques ou non métalliques. Cette méthode est basée sur la résolution du problème de conduction inverse de la chaleur dans l'outil. Les deux aspects que nous avons plus particulièrement abordés concernent d'une part la métrologie mise en œuvre pour la mesure de température dans l'outil et la modélisation qui permet d'exprimer la 


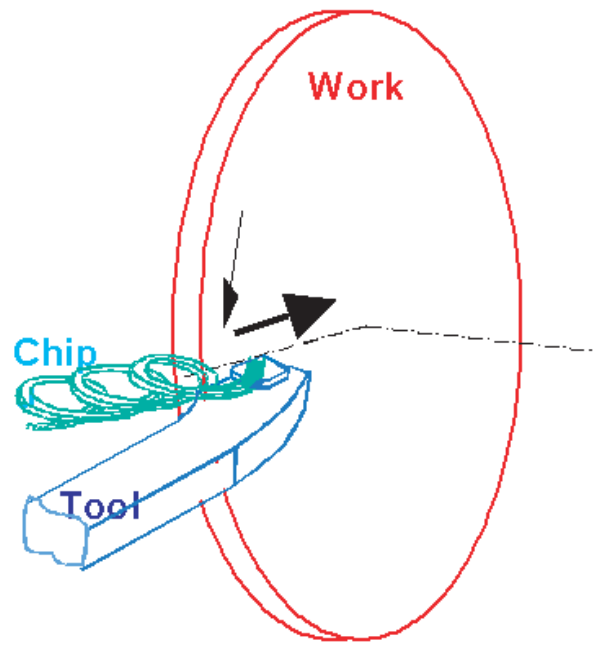

Fig. 5. Représentation schématique du dressage d'un disque par tournage. L'outil se déplace depuis la périphérie du disque jusqu'au centre.

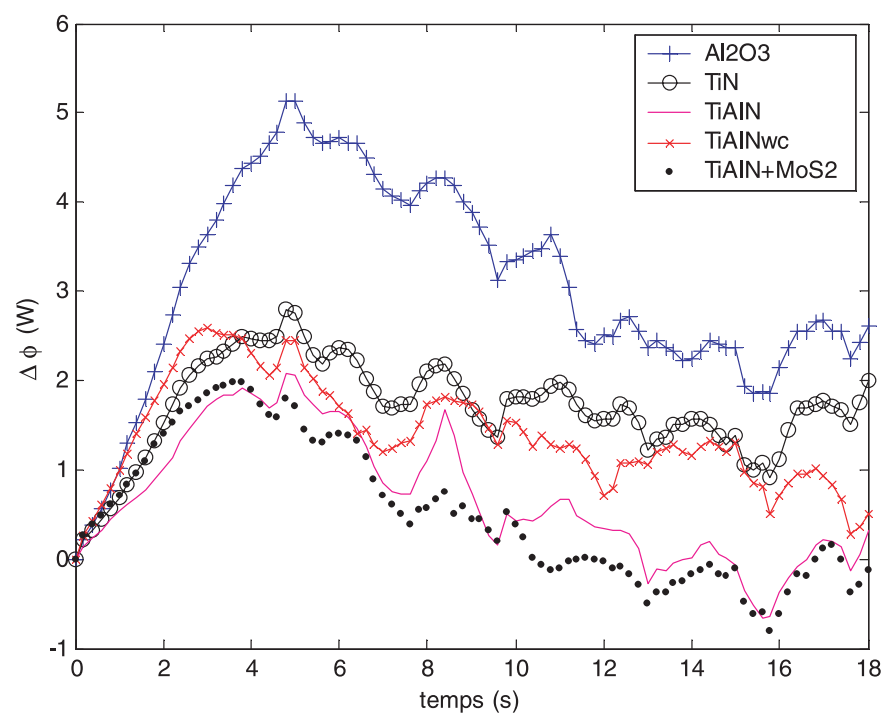

Fig. 6. Estimation du flux de chaleur dans l'outil avec différents revêtements. On représente la comparaison entre le flux dans l'outil non revêtu et celui dans l'outil avec revêtement.

température du capteur en fonction du flux de chaleur dans l'outil.

D'un point de vue de la métrologie, l'utilisation de thermistance est guidée par le rapport signal sur bruit élevé de ce type de composant et le choix d'une période d'échantillonnage des mesures relativement grande devant son temps de réponse. Cette instrumentation permet d'autre part de pouvoir utiliser des contacts glissants classiques pour la mesure de température sur des outils tournants, même à grande vitesse.

La modélisation au sens de l'identification de système non entier trouve ici un domaine d'applications privilégié étant donné la complexité des outils en terme de géométrie et du nombre de constituants. Un avantage important de l'utilisation de système identifié pour la

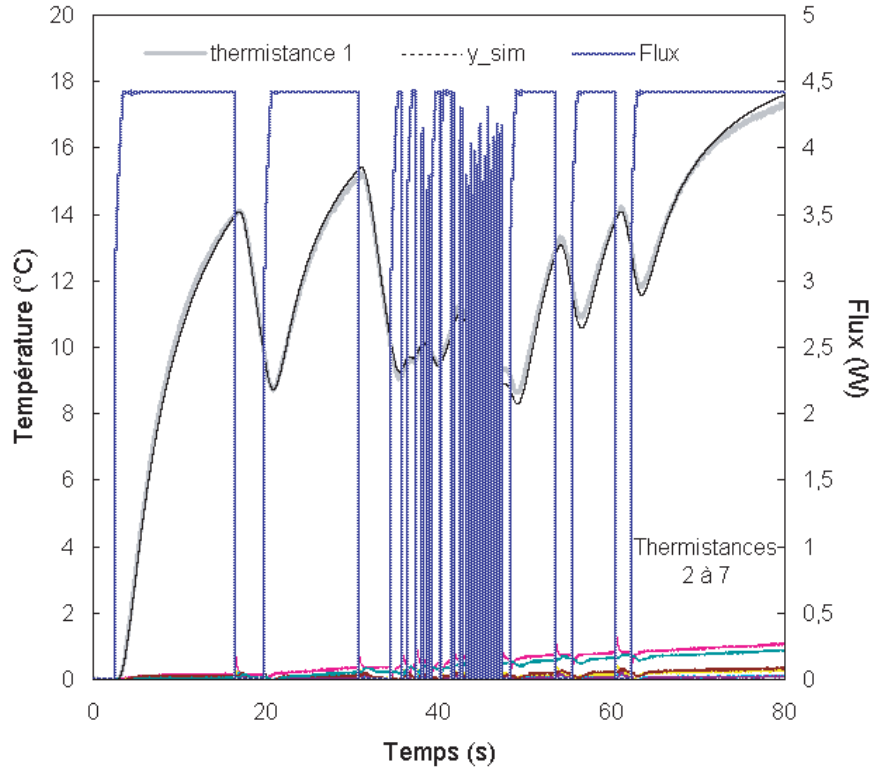

Fig. 7. Essai de caractérisation de l'outil de fraisage. On constate que l'on peut négliger l'influence du flux appliqué sur la plaquette $\mathrm{n}^{\circ} i$ sur les capteurs autres que le $\mathrm{n}^{\circ} i$. On peut alors considérer chaque couple \{plaquette $i$ et thermistance $i$ \} comme un système monovariable.

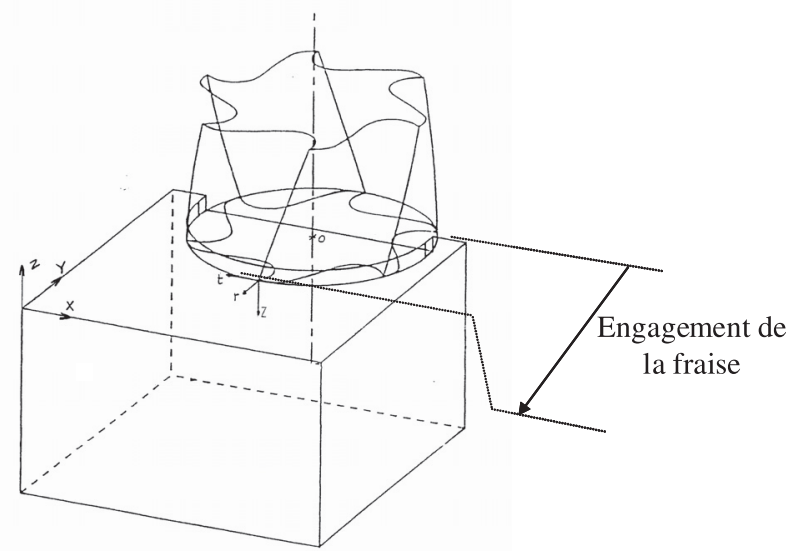

Fig. 8. Déroulement du procédé de surfaçage par fraisage. L'engagement dénote la profondeur de pénétration de la fraise dans la matière. Cet engagement est total lorsqu'il devient égal au rayon de la fraise.

résolution d'un problème inverse est que les paramètres du modèle sont identifiés en positionnant le capteur de température à l'endroit le mieux adapté pour résoudre le problème d'estimation du flux dans l'outil. D'autre part, la position du capteur est invariable lors du passage de la phase de caractérisation de l'outil vers la phase d'estimation du flux durant l'usinage. Il n'existe donc aucune incertitude concernant sa localisation, qui n'a d'ailleurs pas lieu d'être connue précisément. Le deuxième avantage est que le modèle identifié est continu dans le temps. Ceci veut donc dire qu'il est en toute rigueur valable pour n'importe quel échantillonnage temporel. En fait, ce modèle n'est évidemment valable que pour une période 


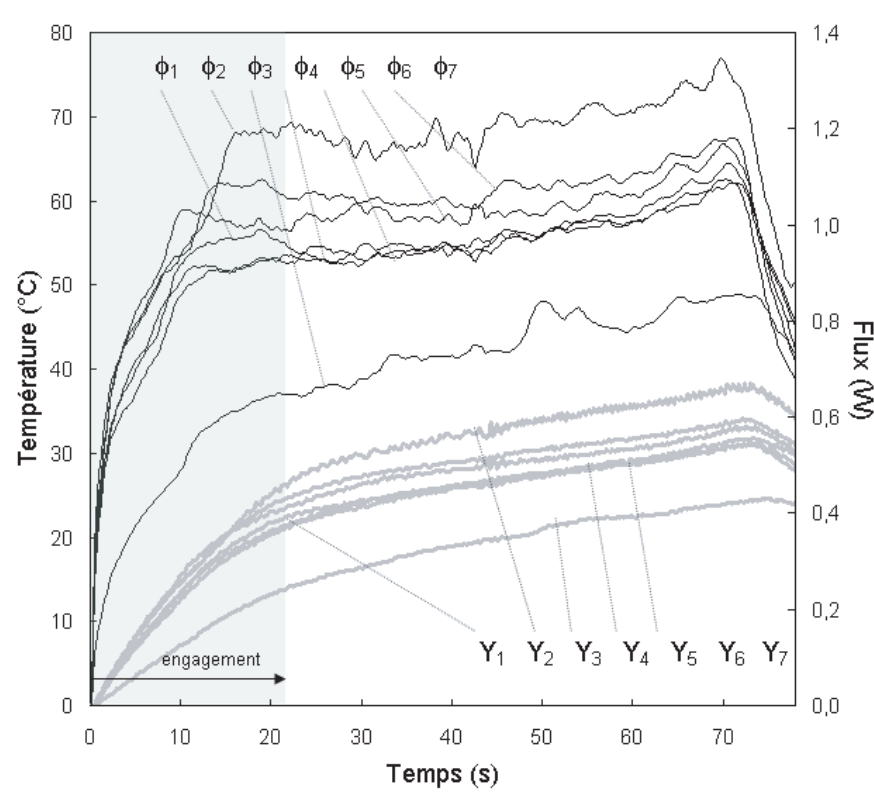

Fig. 9. Évolution de la température de chaque thermistance durant l'essai d'usinage et estimation du flux de chaleur dans chaque plaquette. Le flux de chaleur croît dans chaque plaquette tant que l'engagement de la fraise n'est pas égal au rayon de l'outil de fraise.

d'échantillonnage supérieure ou égale à celle utilisée pour l'identification du modèle. Le troisième avantage est que le modèle identifié comporte peu de paramètres et ceci indépendamment de la dimension spatiale du transfert (1D, 2D ou 3D). Il est donc possible de l'utiliser dans une procédure d'estimation séquentielle et donc d'estimer le flux en temps réel. Enfin, la procédure d'identification prend en compte l'influence spatio-temporelle engendrée par l'utilisation de capteurs, telles que les thermistances, dont les dimensions ne sont pas négligeables devant la distance entre ce capteur et l'arête de coupe.

La principale difficulté de notre approche est que l'on est contraint d'identifier le modèle sur un banc de caractérisation permettant de contrôler et de mesurer le flux imposé sur l'outil. Il est alors primordial de conserver la configuration réelle d'utilisation du système pour ne pas introduire ou occulter des dynamiques inexistantes sur le procédé d'usinage.

Deux applications aux procédés d'usinage par tournage et fraisage ont été présentées. Elles mettent en évidence le rôle joué par les revêtements d'outil et le fonctionnement très différent de chaque plaquette d'un outil de fraisage lors de l'usinage. D'autres applications existent telles que la corrélation entre l'usure d'outil et le flux par exemple. Des résultats ont aussi été obtenus lors de l'usinage par déroulage de matériaux à base de bois et la démarche est facilement transposable au cas du perçage. Néanmoins, dans ce dernier cas, le modèle liant le flux sur chaque bords du foret à la température aux capteurs devient nécessairement multi variable étant donné la petite distance entre les capteurs. Ceci fait l'objet d'un travail en cours de réalisation.

\section{Annexe : algorithme d'identification des paramètres du modèle non entier}

Le modèle non entier liant la température $T_{P}(t)$ au capteur au flux $\phi(t)$ dans l'outil s'écrit :

$$
\sum_{i=0}^{M} a_{i} \mathrm{D}^{i / 2} T_{P}(t)=\sum_{i=0}^{L} b_{i} \mathrm{D}^{i / 2} \phi(t)
$$

En isolant le terme $T_{P}(t)$ dans cette relation, on obtient l'expression du modèle sous la forme de la régression linéaire suivante :

$$
T_{P}(t)=\mathbf{h}(t) \boldsymbol{\theta}
$$

avec :

$$
\begin{aligned}
& \mathbf{h}(t)=\left[\begin{array}{llll}
-\mathrm{D}^{0,5} T_{P}(t) & \cdot-\mathrm{D}^{0,5 M} T_{P}(t) \phi(t) & \cdot \mathrm{D}^{0,5 L} \phi(t)
\end{array}\right] \\
& \text { et } \boldsymbol{\theta}=\left[\begin{array}{lllll}
a_{1} & \cdot & a_{M} & b_{0} & \cdot b_{L}
\end{array}\right]^{T}
\end{aligned}
$$

La mesure de température $Y_{P}(t)$ au point $P$ est entachée d'une erreur $e(t)$, telle que :

$$
Y_{P}(t)=T_{P}(t)+e(t)
$$

En remplaçant la valeur de $T_{P}(t)$ par sa mesure dans la relation (10), on obtient :

$$
Y_{P}(t)=\mathbf{h}^{*}(t) \boldsymbol{\theta}+\varepsilon(t)
$$

Dans cette relation $\varepsilon(t)$ désigne le résidu, encore appelé erreur d'équation, défini par :

$$
\varepsilon(t)=\sum_{i=0}^{M} a_{i} \mathrm{D}^{0,5 i} e(t), a_{0}=1
$$

et :

$$
\mathbf{h}^{*}(t)=\left[-\mathrm{D}^{0,5} Y_{P}(t) \cdot-\mathrm{D}^{0,5 M} Y_{P}(t) \phi(t) \cdot \mathrm{D}^{0,5 L} \phi(t)\right]
$$

La construction de la matrice de régression $\mathbf{h}^{*}(t)$ nécessite l'évaluation numérique des dérivées successives de $\phi(t)$ et de $Y_{P}(t)$. Ce calcul est réalisé en utilisant l'approximation de Grünwald, qui ne conduit à la valeur exacte de la dérivée que lorsque $\Delta t \rightarrow 0$, et qui est définie par :

$$
\begin{aligned}
\mathrm{D}^{\nu} f(t)=\frac{1}{\Delta t^{\nu}} \sum_{k=0}^{K}(-1)^{k}\left(\begin{array}{l}
\nu \\
k
\end{array}\right) f(( & (K-k) \Delta t), \\
\nu \in \mathcal{R}, t & =K \Delta t
\end{aligned}
$$

avec :

$$
\left(\begin{array}{l}
\nu \\
k
\end{array}\right)=\frac{\nu(\nu-1) \ldots(\nu-k+1)}{k !}
$$

L'erreur de mesure contenue dans les données expérimentales est amplifiée par le calcul des dérivées, deux solutions sont alors envisageables. 
Première solution : on applique un filtre sur les valeurs d'entrée et de sortie tel que :

$$
\begin{aligned}
& Y_{P, f}(t)=\int_{0}^{t} h_{f}(\tau) Y_{P}(t-\tau) \mathrm{d} \tau \\
& \text { et } \phi_{f}(t)=\int_{0}^{t} h_{f}(\tau) \phi(t-\tau) \mathrm{d} \tau
\end{aligned}
$$

$h_{f}(t)$ désigne la réponse impulsionnelle du filtre passe-bas défini, dans le domaine de la variable de Laplace, par sa fonction de transfert :

$$
\begin{aligned}
\bar{h}_{f}(s)= & \frac{1}{\left(\left(\frac{s}{\omega_{f}}\right)^{0,5}+1\right)^{N_{f}}}= \\
& \omega_{f}^{0,5 N_{\mathrm{f}}} \times\left(s^{0,5 N_{\mathrm{f}}}+\mathrm{C}_{1}^{N_{\mathrm{f}}} \omega_{f}^{0,5} s^{0,5\left(N_{\mathrm{f}}-1\right)}\right. \\
& \left.+\ldots+\mathrm{C}_{N_{\mathrm{f}}-1}^{N_{\mathrm{f}}} \omega_{f}^{0,5\left(N_{\mathrm{f}}-1\right)} s^{0,5}+\omega_{f}^{0,5 N_{\mathrm{f}}}\right)^{-1}
\end{aligned}
$$

Cette fonction est une généralisation du filtre de Poisson classique, que nous désignons par filtre de Poisson fractionnaire. La fréquence de coupure $\omega_{f}$ sera égale à la fréquence de Nyquist pour la période d'échantillonnage des mesures choisie, soit : $\omega_{f}=2 / \Delta t$. On commet une erreur de calcul moins importante en réalisant en une seule fois le calcul des dérivées des données filtrées. Nous définissons pour cela le vecteur des données d'entrée et de sortie filtrées :

$$
\begin{aligned}
\mathbf{x}=\left[\mathrm{D}^{\left(N_{\mathrm{f}}-1\right) 0,5} q_{f}(t), \mathrm{D}^{\left(N_{\mathrm{f}}-2\right) 0,5} q_{f}(t), \ldots,\right. & \\
& \left.\mathrm{D}^{0,5} q_{f}(t), q_{f}(t)\right]^{T}
\end{aligned}
$$

où $q_{f}(t)$ représente aussi bien $Y_{P, f}(t)$ que $\phi_{f}(t)$. On montre alors que la relation :

$$
q_{f}(t)=\int_{0}^{t} L^{-1}\left[\bar{h}_{f}(s)\right]_{\tau} q(t-\tau) \mathrm{d} \tau
$$

se met sous la forme du modèle d'état :

$$
\begin{aligned}
& \mathrm{D}^{0,5} \mathbf{x}(t)= \\
& \left.\qquad \begin{array}{ccccc}
-\mathrm{C}_{1}^{N_{\mathrm{f}}} \omega_{f}^{0,5} & -\mathrm{C}_{2}^{N_{\mathrm{f}}} \omega_{f} & \cdot \mathrm{C}_{N_{\mathrm{f}}-1}^{N_{\mathrm{f}}} \omega_{f}^{0,5\left(N_{\mathrm{f}}-1\right)} & \omega_{f}^{0,5 N_{\mathrm{f}}} \\
1 & 0 & \cdot & \cdot & 0 \\
0 & \cdot & 0 & \cdot & \cdot \\
. & \cdot & . & 0 & . \\
0 & \cdot & 0 & 1 & 0
\end{array}\right] \mathbf{x}(t) \\
& +\left[\begin{array}{c}
\omega_{f}^{0,5 N_{\mathrm{f}}} \\
0 \\
\cdot \\
\cdot \\
0
\end{array}\right] z(t) \quad(22)
\end{aligned}
$$

En simulant la réponse de ce modèle, nous obtenons ainsi les dérivées fractionnaires des données filtrées.
Après filtrage, l'équation de régression linéaire (13) devient :

$$
Y_{P, f}(t)=\mathbf{h}_{f}^{*}(t) \boldsymbol{\theta}+p(t)
$$

avec :

$\mathbf{h}_{f}^{*}(t)=$

$$
\left[-\mathrm{D}^{0,5} Y_{P, f}(t) \cdot-\mathrm{D}^{0,5 M} Y_{P, f}(t) \phi_{f}(t) \cdot \mathrm{D}^{0,5 L} \phi_{f}(t)\right]
$$

et $p(t)=\int_{0}^{t} h_{f}(\tau) \varepsilon(t-\tau) \mathrm{d} \tau$ définit l'erreur de prédiction filtrée.

En considérant $K$ mesures successives de la température et du flux avec la période d'échantillonnage $\Delta t$, l'équation (23) devient :

$$
\mathbf{Y}_{K}=\mathbf{H} \boldsymbol{\theta}+\mathbf{P}_{K}
$$

avec :

$$
\begin{gathered}
\mathbf{H}=\left[\begin{array}{c}
\mathbf{h}_{f}^{*}(t) \\
\mathbf{h}_{f}^{*}(t+\Delta t) \\
\cdot \\
\mathbf{h}_{f}^{*}(t+K \Delta t)
\end{array}\right], \mathbf{Y}_{K}=\left[\begin{array}{c}
Y_{P, f}(t) \\
Y_{P, f}(t+\Delta t) \\
\cdot \\
Y_{P, f}(t+K \Delta t)
\end{array}\right] \\
\text { et } \mathbf{P}_{K}=\left[\begin{array}{c}
p(t) \\
p(t+\Delta t) \\
\cdot \\
p(t+K \Delta t)
\end{array}\right]
\end{gathered}
$$

Ainsi, l'estimation de $\boldsymbol{\theta}$ au sens des moindres carrés linéaires qui minimise la quantité $J=\mathbf{P}_{K}^{T} \mathbf{P}_{K}$ est donnée par :

$$
\boldsymbol{\theta}=\left(\mathbf{H}^{T} \mathbf{H}\right)^{-1} \mathbf{H}^{T} \mathbf{Y}_{K}
$$

Cet estimateur est non biaisé, c'est-à-dire que sa moyenne sur toutes les données possibles correspond à la vraie valeur des paramètres, si et seulement si $E\left[\left(\mathbf{H}^{T} \mathbf{H}\right)^{-1} \mathbf{H}^{T} \mathbf{P}_{K}=0\right.$. Ce sera le cas si $\mathbf{P}_{K}$ est à moyenne nulle et non corrélé avec $\mathbf{H}$. Or, la relation (14) montre que $\mathbf{H}$ est corrélé avec $\mathbf{P}_{K}$. L'estimateur au sens des moindres carrés linéaires comporte donc un biais. Pour résoudre ce problème, nous utilisons la méthode des variables instrumentales qui consiste à modifier l'équation de régression (25) en multipliant chaque terme par $\mathbf{Z}_{K}$, dont les éléments sont les instruments, tels que l'on ait : $E\left[\mathbf{Z}_{K} \mathbf{P}_{K}\right]=0$. Pour déterminer $\mathbf{Z}_{K}$, la solution la plus simple consiste à remplacer dans $\mathbf{H}$ les éléments susceptibles d'être corrélés avec $\mathbf{P}_{K}$ par les résultats d'une simulation du modèle à une itération précédente. On obtient ainsi la procédure itérative suivante :

$$
\boldsymbol{\theta}_{k+1}=\left(\mathbf{H}_{V I}^{t}\left(\boldsymbol{\theta}_{k}\right) \mathbf{H}\right)^{-1} \mathbf{H}_{V I}^{t}\left(\boldsymbol{\theta}_{k}\right) \mathbf{Y}_{K}
$$


avec $\mathbf{H}$ défini à la relation $(26)$ et :

$$
\begin{array}{r}
\mathbf{H}_{V I}=\left[\begin{array}{c}
\mathbf{h}_{V I}\left(\boldsymbol{\theta}_{k}, t\right) \\
\mathbf{h}_{V I}\left(\boldsymbol{\theta}_{k}, t+\Delta t\right) \\
\cdot \\
\mathbf{h}_{V I}\left(\boldsymbol{\theta}_{k}, t+K \Delta t\right)
\end{array}\right] \\
\text { et } \mathbf{h}_{V I}\left(\boldsymbol{\theta}_{k}, t\right)=\left[\begin{array}{c}
-\mathrm{D}^{0,5} T_{f}\left(\boldsymbol{\theta}_{k}, t\right) \\
\cdot \\
-\mathrm{D}^{0,5 M} T_{f}\left(\boldsymbol{\theta}_{k}, t\right) \\
\phi_{f}(t) \\
\cdot \\
\mathrm{D}^{0,5 L} \phi_{f}(t)
\end{array}\right]{ }^{T}
\end{array}
$$

Le vecteur des paramètres initiaux est obtenu à partir de l'estimation au sens des moindres carrés linéaires de la relation (27). Les itérations stoppent lorsque l'écart sur les paramètres entre deux itérations devient inférieur à une valeur fixée préalablement. Les domaines de confiance sur les paramètres estimés sont issus du calcul de la matrice de covariance sur le vecteur des paramètres optimaux, obtenus lors de la dernière itération. Cette matrice revêt une expression complexe et une approximation est classiquement donnée par :

$$
\operatorname{cov}\left[\boldsymbol{\theta}_{\text {opt }}\right]=\left(\mathbf{H}_{V I}^{T} \mathbf{H}\right)^{-1} s^{2}
$$

avec :

$$
s=\frac{J\left(\boldsymbol{\theta}_{\text {opt }}\right)}{K-n}, n=L+M-1
$$

On obtient alors l'écart type sur chaque paramètre $\theta_{i}$ par :

$$
\sigma\left(\theta_{i}\right)=\sqrt{\operatorname{cov}\left(\boldsymbol{\theta}_{\text {opt }}\right)_{i, i}}
$$

On doit néanmoins garder à l'esprit que le caractère non biaisé d'un estimateur n'est pas forcément une qualité en regard des paramètres estimés. En effet, il est parfois préférable d'accepter un certain biais, si cela conduit à une réduction de variance $J(\theta)$ plus importante. L'évolution de cette variance doit donc être considérée comme un critère d'arrêt des itérations dans la méthode des variables instrumentales.

Deuxième solution : On utilise le modèle basé sur l'intégration des grandeurs mesurées, qui va donc consister à seulement inverser le signe des ordres de dérivation dans la relation (10). Cela conduit donc à :

$$
\sum_{i=0}^{M} a_{i} D^{-i / 2} T_{P}(t)=\sum_{i=0}^{L} b_{i} D^{-i / 2} \phi(t)
$$

L'estimateur est alors directement donné au travers de la relation (27). Cette solution est très simple à mettre en œuvre mais elle reste limitée à des ordre de dérivation peu élevés car l'amplitude du signal est elle aussi affectée par l'intégration.

\section{Références}

[1] G. Barrow, A review of experimental and theorical techniques for assessing cutting temperatures, Annals of the CIRP 22 (1973) 203-211

[2] H. Shore, Thermoelectric measurement of cutting tool temperatures, J. Washington Academy of Sciences 15 (1925) 85-88

[3] E.G. Herbert, The measurement of cutting, Proc. Inst. Mech. Engr. 1 (1926) 289-329

[4] D.A. Stephenson, Tool-work thermocouple temperature measurements - Theory and implementation issues, Journal of Engineering for industry 115 (1993) 432-437

[5] M. Lipman, B. Nevis, G. Kane, A remote sensor method for determining average tool-chip interface temperatures in metal cutting, Journal of Engineering for Industry 89 (1967) 333-338

[6] M.P. Groover, G.E. Kane, A continuing study in the determination of temperatures in metal cutting using remote thermocouples, Journal of Engineering for Industry 87 (1971) 603-608

[7] W.D. Yen, P.K. Wright, A remote temperature sensing technique for estimating the cutting interface temperature distribution, Journal of Engineering for Industry 108 (1986) 252-263

[8] D.A. Stephenson, An inverse method for investigating deformation zone temperatures in metal cutting, Journal of Engineering for Industry 113 (1991) 129-136

[9] J. Lin, Inverse estimation of tool-work interface temperature in end milling, Int. J. Mach. Tools Manufact. 35 (1995) $751-760$

[10] T.I. El-Wardany, E. Mohammed, M.A. Elbestawi, Cutting temperature of ceramic tools in high speed machining of difficult-to-cut materials, Int. J. Mach. Tools Manufact. 36 (1996) 611-634

[11] W.-C. Chen, C.-C. Tsao, P.-W. Liang, Determination of temperature distributions on the rake face of cutting tools using a remote method, Int. Comm. Heat Mass Transfer 24 (1997) 161-170

[12] P. Kwon, T. Schiemann, R. Kountanya, An inverse estimation scheme to measure steady-state tool-chip interface temperatures using an infrared camera, Int. J. Mach. Tools Manufact. 41 (2001) 1015-1030

[13] L. Olson, R. Throne, Estimation of tool-chip interface temperatures for on-line monitoring : an inverse problem approach, Inverse Problems in Engineering 9 (2001) 367388

[14] F.R.S. Lima, A. Machado, G. Guimarães, S. Guth, Numerical and experimental simulation for heat flux and cutting temperature estimation using three dimensional inverse heat conduction technique, Inverse Problems in Engineering 8 (2000) 675-676

[15] J.-L. Battaglia, L. Le Lay, J.-C. Batsale, A. Oustaloup, O. Cois, Heat flux estimation through inverted non integer identification models, Int. J. Thermal Science 39 (2000) 374-389

[16] J.-L. Battaglia, O. Cois, L. Puigsegur, A. Oustaloup, Solving an inverse heat conduction problem using a noninteger identified model, Int. J. Heat Mass Transfer 14 (2000) 2671-2680

[17] L. Puigsegur, J.-L. Battaglia, J.-C. Batsale, O. Cois, Identification de modèles du transfert de chaleur par 
conduction dans un système. Utilisation d'une structure à dérivées fractionnaires. Congrès SFT 2001, Ed. Elsevier, pp. $745-750$

[18] J.V. Beck, B. Blackwell, C.R. ST. Clair, Inverse Heat conduction, A Wiley-Interscience Publication, 1985

[19] L. Ljung, System identification : theory for the user, Prentice Hall, 1987

[20] T. Söderstrom, P. Stoïca, System identification, Prentice Hall, 1989

[21] J.-L. Battaglia, J.-C. Batsale, Estimation of heat flux and temperature in a tool during turning, Inverse Problems in Engineering 8 (2000) 435-456

[22] K.S. Miller, B. Ross, An introduction to the fractional calculus and fractional differential equations, A WileyInterscience Publication, 1993
[23] K.B. Oldham, J. Spanier, The fractional calculus, Academic Press, New York and London, 1974

[24] A. Oustaloup., La dérivation non entière : théorie, synthèse et applications, Ed. Hermès, Paris, 1995

[25] S.G. Samko, A.A. Kilbas, O.I. Marichev, Fractional Integrals and Derivatives. Theory and applications, Gordon and Breach, Amsterdam, 1993

[26] M. Broussely, Réduction de modèles thermiques par la théorie des réseaux, application à la surveillance d'une machine asynchrone par couplage d'un modèle thermique réduit avec un schéma équivalent électrique, Thèse de l'université de Poitiers, 2000

[27] P.C. Young, Parameter estimation for continuous-time models - a survey, Automatica 17 (1981) 23-29 